\title{
GC-MS analysis of essential oil of Paeonia suffruticosa Andrews from ZhaoFen and RouFurong flowers in China
}

\author{
Weiping Yin*, Wenlu Duan, Pu Liu, Ruixue Deng, Yunlai Ren, Shuang Zhao \\ School of Chemical Engineering and Pharmaceutics, Henan University of Science and Technology, Luoyang, China; \\ *Corresponding Author: yinwp@mail.haust.edu.cn
}

Received 16 June 2012; revise 20 July 2012; accepted 30 July 2012

\begin{abstract}
The flowers of ZhaoFen and RouFurong may contain essential oils with natural aromatic ingredients. In the present work, the chemical compositions of essential oil of Paeonia suffruticosa Andrews from the flowers of ZhaoFen and RouFu-rong grown only in China were investigated by GC-MS analysis. The results indicate that there are 27 constituents in ZhaoFen and 29 constituents in RouFurong, which account for $96.04 \%$ and $95.90 \%$ of the oils of ZhaoFen and RouFurong, respectively. The major components of the essential oils are characterrized by oxygenated terpenols, and their content is, respectively, $85.06 \%$ and $83.47 \%$. The essential oil of Paeonia suffruticosa Andrews was reported for the first time on the aerial parts.
\end{abstract}

Keywords: Chemical Composition; Essential Oil; The Flowers of Paeonia suffruticosa Andrews; GC-MS Analysis; Oxygenated Terpenol

\section{INTRODUCTION}

The genus peony, classified as the Paeonia family, includes 35 species and is distributed in the temperate zone of the west of Asia, Europe and North America [1,2]. There are 14 species in China and they spread in the west hill country of central northeast $[3,4]$. Paeonol $(2$ '-hydroxy-4'-methoxyacetophenone) is a Mincom-potent from the root barks of Peoria suffruticosa Andrews, and has been applied in the treatment of blood stasis, allergic rhinitis, hypertensions and inflammatory diseases etc [5-7]. The ZhaoFen and RouFurong flowers of Paeonia suffruticosa Andrews, grown only in the midwest region of China, is one of the Chinese traditional famous flowers with a characteristic aromatic odor [8]. In recent years, they are becoming increasingly popular as natu- rally occurring spices reagent in folk. However, very few information on their essential oils and components is available in the literature up to now. In the present work, therefore, a phytochemical investigation on the essential oil of Paeonia suffruticosa Andrews from the flowers of ZhaoFen and RouFurong was reported for the first time.

\section{MATERIAL AND METHODS}

The essential oil was extracted from fresh flowers of ZhaoFen and RouFurong (Paeonia suffruticosa) as the pale yellow oil in $0.005 \%$ and $0.004 \%$ yields, respectively.

Two species of Paeonia flowers were collected from Luoyang city of China at the flowering stage during April-May 2011 and processed immediately after harvest. A cohobating by the semi-industrial stainless steel distilling apparatus with recirculation of the condensed water was used. The samples were distilled for three hours and the oils extracted by n-hexane were dried with anhydrous sodium sulphate, and then stored in dark vials at $4^{\circ} \mathrm{C}[9]$.

The samples were chromatographed using an Agilent 6890 GC equipped with an Agilent 5973 Mass selective detector, a 7694 autosampler and a split-splitless injector. The GC was linked to an MS ChemStation HP vs. C.00.07. A DB5MS fused silica column $(30 \mathrm{~m} \times 0.25$ $\mathrm{mm}$, film thickness $0.25 \mu \mathrm{m})$. The injector and interface were operated at $150^{\circ} \mathrm{C}$ and $280^{\circ} \mathrm{C}$, respectively. The oven temperature was programmed to raise from $60^{\circ} \mathrm{C}$ to $180^{\circ} \mathrm{C}\left(3^{\circ} \mathrm{C} / \mathrm{min}\right)$, and held for $15 \mathrm{~min}$. Helium was used as the carrier gas at $0.9 \mathrm{ml} / \mathrm{min}$, and the sample $(1 \mu \mathrm{l})$ was injected in the split MS conditions: ionization voltage of $70 \mathrm{eV}$, scan rate of $1.6 \mathrm{scan} / \mathrm{sec}$, mass range of 50 -500 , and ion source temperature of $180^{\circ} \mathrm{C}$.

\section{RESULTS AND DISCUSSIONS}

The components of essential oils were identified by comparing their relative retention times and mass spectra 
with those of authentic samples (analytical standards from Aldrich). Sample solutions were prepared in nhexane (GC grade, Merck) at 1.0\% (w/w). The results of the GC and GC-MS analyses were listed in Tables 1 and 2 , in which the compounds were shown in the order of their elution time on the column. Chemical composition

Table 1. Chemical composition of the essential oil from the ZhaoFen flower.

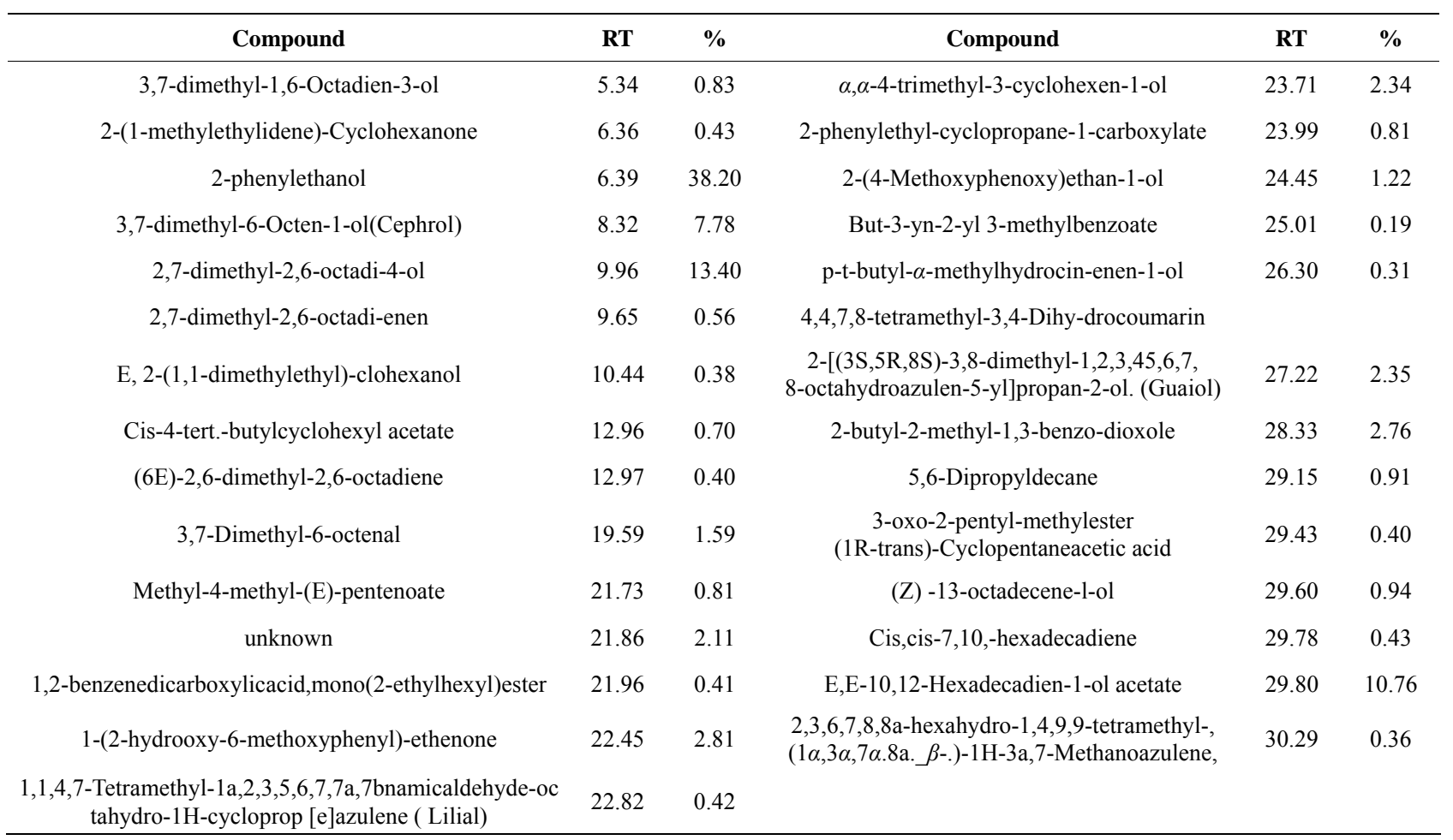

Identification method: RI, MS; Percentage $\left(\%^{b}\right)$ was calculated from flame ionization detector (FID). RT (Retention time).

Table 2. Chemical composition of the essential oil from the RouFurong flower.

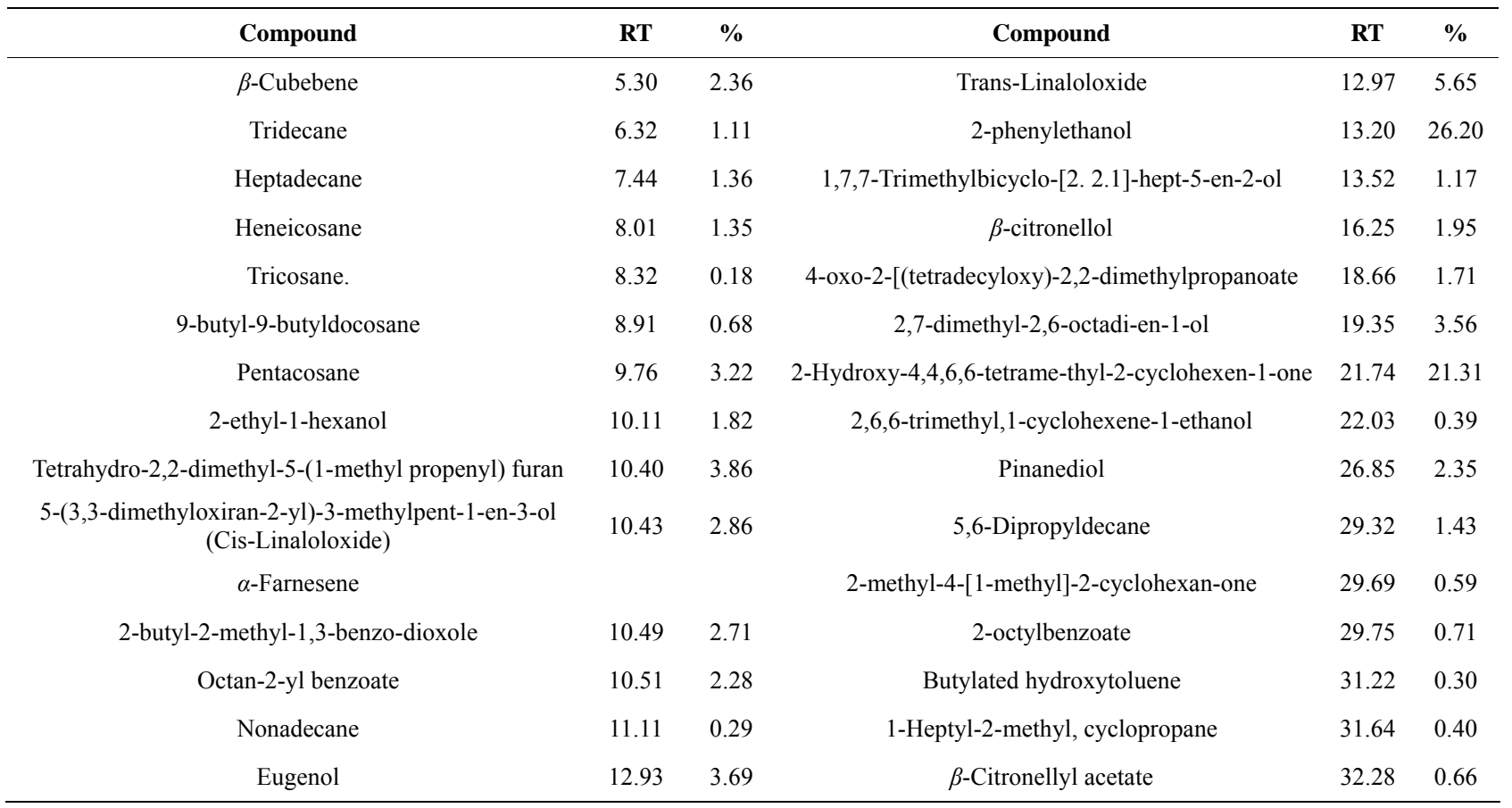

Identification method: RI, MS; Percentage $\left(\%^{b}\right)$ was calculated from flame ionization detector (FID). RT (Retention time). 
of essential oil of the ZhaoFen flowers was presented in Table 1, and the GC-MS analysis leads to the identification of 27 constituents, accounting for $96.04 \%$ of the oil of ZhaoFen. Chemical composition of essential oil of the RouFurong flowers was displayed in Table 2, and the GC-MS analysis suggests that there are 29 constituents, which accounts for $95.90 \%$ of the oil of RouFurong.

As shown in Table 1, 2-phenylethanol (38.20\%) [9] and 2,7-dimethyl-2,6-octadi-4-ol (13.40\%) were the main compounds of ZhaoFen, and the key components with a floral fragrance properties are E, E-10, 12-hexadecadien-1-ol (10.76\%), 3,7-dimethyl-6-octen-1-ol (Cephrol, $7.78 \%$ ) [10] and 2-[(3S,5R,8S)-3,8-dimethyl-1,2,3,4,5,6, 7,8-octahydroazulen-5-yl] propan-2-ol (Guaiol, 2.35\%).

It can be seen from Table 2 that 2-phenylethanol (26.20\%) and 2,7-dimethyl-2,6-octadi-4-ol (3.56\%) are the main compounds of RouFurong, and 2-hydroxy-4,4,6, 6-tetramethyl-2-cyclohexen-1-one (21.31\%), tetrahydro-2, 2-dimethyl-5-(1-methylpropenyl) furan (3.86\%), 2-butyl2-methyl-1,3-benzo-dioxole (2.71\%), eugenol (3.69\%) [11] and translinaloloxide (5.65\%) are the key components of aromatic odor of this species. From Tables 1 and 2 we can see that compounds of both the essential oils could be classified into four categories in total based on their molecular structures: oxygenated terpenols account for $85.06 \%$ and $83.47 \%$, monoterpene hydrocarbons $0.91 \%$ and $9.33 \%$, diterpene hydrocarbons $1.21 \%$ and $2.66 \%$, sesquiterpene hydrocarbons $4.51 \%$ and $4.36 \%$, respectively, in ZhaoFen and RouFurong. Apparently, the content of monoterpene hydrocarbons is quite different in both of the flowers.

\section{CONCLUSION}

The results of the present study suggest that the essential oil can be used as natural spices and would be a potential source for alternative peony essence. Further investigation is needed to confirm their difference in flavor types and in solubility of the essential oil in different matrixes that will likely lead to study the structure-activity relationship between the major components in the essential oil and the characteristic aromatic odor.

\section{ACKNOWLEDGEMENTS}

This research was supported by a grant (No. 2008306701) from
High-Tech R\&D program of Henan province, People's Republic of China.

\section{REFERENCES}

[1] Fang, Y.Y. (1989) Flora of Zhejiang. Science and Technology Publishing House, Hangzhou, 5, 288-289.

[2] Tamura, M. (2007) The families and genera of vascular plants. Springer-Verlag, Berlin, Heidelberg, 265-269.

[3] An, R.B., Kim, H.C., Lee, S.H., Jeong, G.S., Sohn, D.H., Park, H., Kwon, D.Y., Lee, J.H. and Kin, Y.C. (2006) A new monoterpene glycoside and antibacterial monoterpene glycosides from Paeonia suffruticosa. Archives of Pharmacal Research, 29, 815-820. doi:10.1007/BF02973899

[4] Ryu, G., Park, E.K. Joo, J.H., Lee, B.H., Chol, B.W., Jung, D.S. and Lee, N.H. (2001) A new antioxidant monoterpene glycoside, alphabenzoyloxypaeoniflorin from Paeonia suffruticosa. Archives of Pharmacal Research, 24, 105-108. doi:10.1007/BF02976476

[5] Jian, L.Y., Yang, Y.H., Xu, Y.H. and He, Z.G. (2005) The solubilization effect of 2-hydroxypropyl-beta-cyclodextrin on paeonolum. China Journal of Chinese Materia Medica, 30, 1154-1156.

[6] Wang, Q.F., Fan, X.W., Xu, L. and Yao, Y. (2007) Preparation of inclusion complex of paeonol and $\beta$-cyclodextrin by sealed-control temperature method. China Journal of Chinese Materia Medica, 32, 218-221.

[7] Boo1, K.H., Lee, D., Woo, J.K., Ko, S.H., Jeong, E.H., Hong, Q. Riu, K.Z. and Lee, D.S. (2011) Anti-bacterial and anti-viral activity of extracts from Paeonia lactiflora roots. Journal of the Korean Society for Applied Biological Chemistry, 54, 132-135.

[8] Wu, C.Y. and Li, X.W. (1977) Flora reipublicae popularis sinicae. Science Press, Beijing, 66, 287-292.

[9] Godefroot, M., Sandra, P. and Verzele, M. (1981) New method for quantitative essential oil analysis. Journal of Chromatography A, 203, 325-335.

[10] Ren, G., Zhao, Y.P., Shao, F. and Liu, R.H. (2011) Composition and antimicrobial activity of the essential oil of Mosla hangchowensis endemic to China. Chemistry of Natural Compounds, 47, 128-129. doi:10.1007/s10600-011-9855-y

[11] Johnson, C.B., Kirby, J., Naxakis, G. and Pearson, S. (1999) Substantial UV-B-mediated induction of essential oils in sweet basil, Ocimum basilicum L. Phytochemistry, 51, 507-510. doi:10.1016/S0031-9422(98)00767-5 